\title{
Short Pitch Structures in Smectics with Interactions over more than Two Layers
}

\author{
MOJCA ČEPIČ ${ }^{a, b}$, BARBARA ROVŠEK ${ }^{a, c}$, BOŠTJAN ŽEKŠ ${ }^{a, d}$ \\ ${ }^{a}$ J. Stefan Institute, Jamova $39 ;{ }^{b}$ Faculty of Education, Kardeljeva \\ pl. 16; ${ }^{c}$ Faculty of Mathematics and Physics, Jadranska 19; ${ }^{d}$ Insti- \\ tute of Biophysics, Medical Faculty, Lipičeva 2, all 1000 Ljubljana, \\ Slovenia;
}

Phenomenological model of chiral polar smectics is introduced with interactions up to the fourth neighboring layers. The minimization of the free energy gives three stable structures: the ferroelectric $\mathrm{Sm} \mathrm{C}^{*}$ phase, the antiferroelectric $\mathrm{Sm} \mathrm{C}_{A}^{*}$ phase and the hellicoidally modulated structure of the $\mathrm{Sm} \mathrm{C}_{H}^{*}$ phase. The $\mathrm{Sm} \mathrm{C}_{H}^{*}$ phase can be recognized as the $\mathrm{Sm} \mathrm{C}_{\alpha}^{*}$ phase below $\mathrm{Sm} \mathrm{A}$ phase and as the $\mathrm{Sm} \mathrm{C}_{F I 2}^{*}$ phase or as the $\mathrm{Sm} \mathrm{C}_{F I 1}^{*}$ phase when appearing between the $\mathrm{Sm} \mathrm{C}^{*}$ phase and the $\mathrm{Sm} \mathrm{C}_{A}^{*}$ phase. Stability of these phases is analyzed and the phase diagram in the space of model parameters is presented.

Keywords: antiferroelectric liquid crystals; interlayer interactions; hellicoidal modulations.

\section{INTRODUCTION}

Recent observation of various subphases in the thiobenzoate antiferroelectric liquid crystal 10OTBBB1M7 performed by polarized 
x-ray scattering [1] has revealed the structure of the two phases which are stable in the temperature region between the ferroelectric $\mathrm{Sm} \mathrm{C}^{*}$ phase and the antiferroelectric $\mathrm{Sm} \mathrm{C}_{A}^{*}$ phase. The studied material has the following phase sequence with decreasing temperature $\mathrm{Sm} \mathrm{A} \leftrightarrow \mathrm{Sm} \mathrm{C}_{\alpha}^{*} \leftrightarrow \mathrm{Sm} \mathrm{C}^{*} \leftrightarrow \mathrm{Sm} \mathrm{C}_{F I 2}^{*} \leftrightarrow \mathrm{Sm} \mathrm{C}_{F I 1}^{*}$ $\leftrightarrow \quad \mathrm{Sm} \mathrm{C}_{A}^{*}$ with first order transitions between the phases. The phase sequence in this material is general for antiferroelectric liquid crystals. In some materials some of the phases are missing [2].

The $\mathrm{Sm} \mathrm{C}_{\alpha}^{*}$ phase which appears directly below the $\mathrm{Sm} \mathrm{A}$ phase is hellicoidally modulated over few layers and its period is in general not commensurate with the layer thickness [3]. The polarized x-ray scattering [1] has confirmed its structure, which is also consistent with indirect ellipsometric observations $[4,5]$.

The $\mathrm{Sm} \mathrm{C}_{F I 2}^{*}$ phase appears directly below the $\mathrm{Sm} \mathrm{C}^{*}$ phase. It is modulated over approximately four smectic layers. Only the structures where the molecular tilts are not in a single plane are consistent with the experimental data. The simplest structure consistent with experiments is presented within the clock model where tilts of the molecules are constant in the magnitude but differ in direction from the layer to the layer for an approximate angle $\pm 90^{\circ}$. The phase difference has only one sign, and has opposite signs in the two enantiomers of the same materials. The hellicoidal modulation in these systems has a very short pitch of approximately four layers.

The $\mathrm{Sm} \mathrm{C}_{F I 1}^{*}$ phase which appears below the $\mathrm{Sm} \mathrm{C}_{F I 2}^{*}$ phase and above the $\mathrm{Sm} \mathrm{C}_{A}^{*}$ phase is hellicoidaly modulated over approximately three layers. Again, in the most simple structure the difference of the tilt direction is approximately $120^{\circ}$. For this phase the typical textures varies with time also in a temperature stabilized sample. Also peaks of the x-ray measurements are much wider than in other phases, which could be the consequence of various defects or fluctuations.

The structures with short helices were theoretically predicted 
in systems with competing interactions between nearest and next nearest neighboring layers [3]. In the extended model of antiferroelectric liquid crystals [6], the $\mathrm{Sm}_{\alpha}^{*}$ phase was predicted as the hellicoidally modulated over few layers only, while the structure between the $\mathrm{Sm} \mathrm{C}^{*}$ and the $\mathrm{Sm} \mathrm{C}_{A}^{*}$ phase consisted of two helices geared into each other for a general angle. The last structure although consistent with various observations [2], has been ruled out by the first direct structural observation [1].

In this paper we analyze the consequences of the interactions which extend over more than two neighboring layers. In order to stabilize structures with three and four layers periodicities, coupling terms of the same range are needed [7]. Therefore we introduce such terms in the expression for the free energy and analyze their influence on the various structures in these systems. We find three different stable structures: the structure with the synclinic tilt or the $\mathrm{Sm} \mathrm{C}^{*}$ phase, the structure with the anticlinic tilt or the $\mathrm{Sm} \mathrm{C}_{A}^{*}$ phase and the hellicoidally modulated structure we called the $\mathrm{Sm} \mathrm{C}_{H}^{*}$ phase. The $\mathrm{Sm} \mathrm{C}_{H}^{*}$ phase with the phase difference $\alpha$ approximatelly $90^{\circ}$ is stable when interactions between nearest neighboring layers are weak and interactions with next nearest neighboring layers favor anticlinic ordering [3]. The structure corresponds to the $\mathrm{Sm} \mathrm{C}_{F I 2}^{*}$ phase. The $\mathrm{Sm} \mathrm{C}_{H}^{*}$ phase with the modulation of approximately three layers corresponds to the $\mathrm{Sm} \mathrm{C}_{F I 1}^{*}$ phase and is stabilized by the interactions with third neighboring layers which favor synclinic tilts. In addition we find that interactions with third and fourth neighboring layers cause first order transition between the $\mathrm{Sm} \mathrm{C} \mathrm{C}^{*}$ and the $\mathrm{Sm} \mathrm{C} \mathrm{C}_{H}^{*}$ phase and between the $\mathrm{Sm} \mathrm{C}_{A}^{*}$ and the $\mathrm{Sm} \mathrm{C}_{H}^{*}$ phase, and can account for the first order transitions between the $\mathrm{Sm} \mathrm{C}_{F I 1}^{*}$ phase and the $\mathrm{Sm} \mathrm{C}_{A}^{*}$ phase or between the $\mathrm{Sm} \mathrm{C} \mathrm{C}_{F I 1}^{*}$ phase and the $\mathrm{Sm} \mathrm{C}^{*}$ phase. For some sets of model parameters two $\mathrm{Sm} \mathrm{C}_{H}^{*}$ phases with two different periods are stable and can account for the first order transition between the $\mathrm{Sm} \mathrm{C}_{F I 1}^{*}$ and 
the $\mathrm{Sm} \mathrm{C}_{F I 2}^{*}$ phase. The results are given in phase diagrams in the model parameter space. Finally, we conclude and discuss some open questions.

\section{FREE ENERGY}

In the system where periodical structures with periods over three or four smectic layers exist, we expect some interactions over the same number of layers. Although it is not reasonable to expect that direct interactions extend over more than two layers layers [8], indirect interactions of longer range are possible due to the flexoelectric interactions [9]. To describe effective direct and indirect interactions we write tilt order parameter in the $j$-th layer as $\vec{\xi}_{j}=<\left\{n_{j, x} n_{j, z}, n_{j, y} n_{j, z}\right\}>=\left\{\xi_{j, x}, \xi_{j, y}\right\}$. Achiral interlayer interactions in their simplest form are given as scalar products between tilt vectors in neighboring layers weighted by the model parameters which give the strength of the interlayer coupling. In this analysis chiral interactions will be neglected. The free energy of the system with interactions up to fourth neighboring layers

$G=G_{0}+\sum_{j} a_{1}\left(\vec{\xi}_{j} \cdot \vec{\xi}_{j+1}\right)+a_{2}\left(\vec{\xi}_{j} \cdot \vec{\xi}_{j+2}\right)+a_{3}\left(\vec{\xi}_{j} \cdot \vec{\xi}_{j+3}\right)+a_{4}\left(\vec{\xi}_{j} \cdot \vec{\xi}_{j+4}\right)$.

In the expression above $G_{0}$ is the part of the free energy which does not depend of the interlayer interactions. Model parameters $a_{1}$ to $a_{4}$ give the strength of interlayer interactions and favor synclinic tilts in interacting layers for their negative signs and anticlinic tilts for their positive signs [3]. Interactions are in general competing. The sign of $a_{1}$ can be either positive or negative $[8,10]$. In this analysis the sign of the $a_{2}$ parameter will be taken as positive since this is expected for the systems with hellicoidal modulations over few layers. To stabilize the structure with the three layer periodicity the sign of the $a_{3}$ has to be negative, the fact which is also in agreement with the 
physical origin of this interaction [11]. The four layer structures are stabilized already for the significant $a_{2}$ model parameter, therefore we consider both signs of the model parameter $a_{4}$.

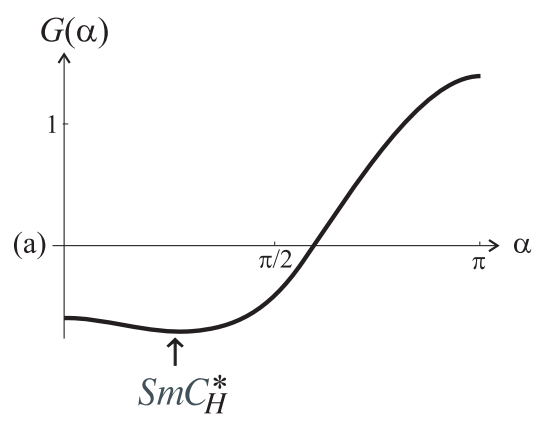

(b)
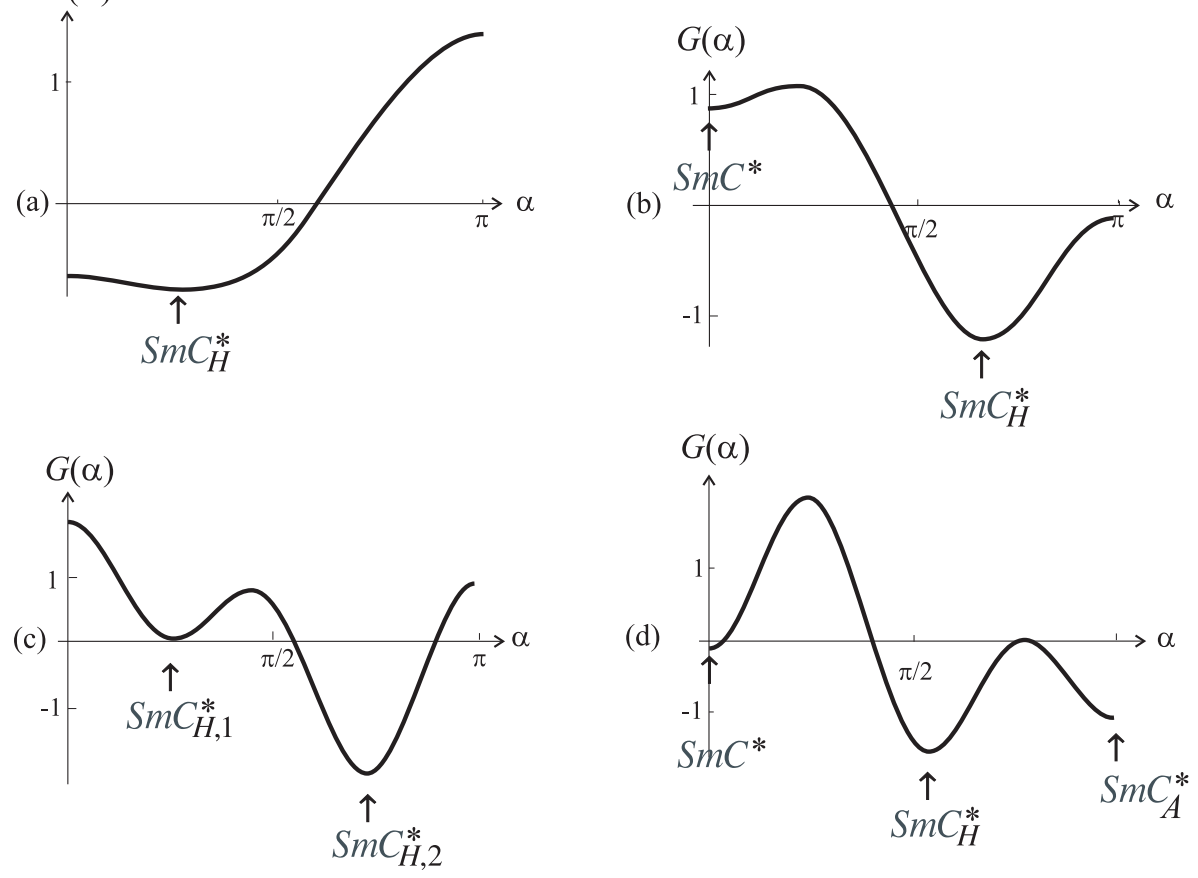

Figure 1: Free energy $G(\alpha)$ for the model parameters: a) $a_{1}=-1.0, a_{2}=$ $0.4, a_{3}=0.0, a_{4}=0.0$; one possible stable phase, b) $a_{1}=1.0, a_{2}=$ $0.4, a_{3}=-0.5, a_{4}=0.0$; two possible stable phases, c) $a_{1}=1.0, a_{2}=$ $0.4, a_{3}=-0.5, a_{4}=1$; two possible stable $\mathrm{Sm} \mathrm{C}_{H}^{*}$ phases, d) $a_{1}=$ $1.0, a_{2}=0.4, a_{3}=-0.5, a_{4}=-1$; three possible stable phases.

To minimize the free energy (Eq. 1) we assume that the tilt varies only in its direction and not in its magnitude and is given by $\theta$. Further, here we analyze only structures of the clock model type, which means that the difference of the tilt directions in neighboring layers $\alpha$ is constant in the magnitude and the sign. Such structures we called the $\mathrm{Sm} \mathrm{C}_{H}^{*}$ phase for a general value of $\alpha$ different from 0 
or $\pi$. We therefore look for the solution of the type

$$
\vec{\xi}_{j}=\theta\{\cos j \alpha, \sin j \alpha\}
$$

where the initial phase angle in the first layer is set to zero which is allowed due to the rotational symmetry. The free energy has now the form

$$
G=G_{0}+\theta^{2}\left(a_{1} \cos \alpha+a_{2} \cos 2 \alpha+a_{3} \cos 3 \alpha+a_{4} \cos 4 \alpha\right) .
$$

Since we are interested only in the periods of structures and not in the tilt magnitude, we have to minimize the free energy only with respect to the phase difference $\alpha$. Derivation with respect to $\alpha$ gives

$$
\left(a_{1}-3 a_{3}+4\left(a_{2}-4 a_{4}\right) \cos \alpha+12 a_{3} \cos ^{2} \alpha+32 a_{4} \cos ^{3} \alpha\right) \sin \alpha=0 .
$$

The solutions of the (Eq. - (1) present locally stable minima providing second derivative with respect to $\alpha$ is positive. For different sets of model parameters we find one, two or three locally stable structures.

In Fig. 1 we present free energy $G(\alpha)$ for a few sets of model parameters. In systems with only nearest and next nearest neighbors interactions, only one stable solution for the phase difference $\alpha$ is possible (Fig. 1a) and corresponds to the $\mathrm{Sm} \mathrm{C}^{*}$ phase, the $\mathrm{Sm} \mathrm{C}_{A}^{*}$ phase or the $\mathrm{Sm} \mathrm{C}_{H}^{*}$ phase. In systems where additional third nearest neighbors interactions are not negligible, the $\mathrm{Sm} \mathrm{C}^{*}$ phase and the $\mathrm{Sm} \mathrm{C}_{H}^{*}$ phase can be stable for the same set of parameters, which gives rise to the first order transition between the two of them (Fig. 1b). If also fourth nearest neighbors interactions are present, they can stabilize either two $\mathrm{Sm} \mathrm{C}_{H}^{*}$ modulated phases (Fig. 1c) or even three phases - the $\mathrm{Sm} \mathrm{C}^{*}$ phase, the $\mathrm{Sm} \mathrm{C}_{A}^{*}$ phase and the $\mathrm{Sm} \mathrm{C}_{H}^{*}$ phase for the same set of model parameters (Fig. 1d). 


\begin{tabular}{|c|c|c|}
\hline the phase & phase angle & properties \\
\hline $\mathrm{SmA}$ & not defined & paraelectric, no ORP \\
\hline $\mathrm{SmC}^{*}$ & $\approx 0$ & ferroelectric, ORP \\
\hline $\mathrm{SmC}_{A}^{*}$ & $\approx \pi$ & antiferroelectric, ORP \\
\hline $\mathrm{SmC}_{\alpha}^{*}$ & general value & mainly paraelectric, no ORP \\
\hline $\mathrm{Sm} \mathrm{C}_{F I 2}^{*}$ & $\approx \pi / 2$ & antiferroelectric, ORP \\
\hline $\mathrm{Sm} \mathrm{C}$ \\
\hline
\end{tabular}

Table 1: Phases and corresponding phase angles $\alpha$. The value of the phase angle is not exactly 0 or $\pi$ in chiral samples. Some macroscopic properties are also given.

\section{PHASE DIAGRAM AND THE STABILITY OF THE PHASES}

From the symmetry point of view, all structures with various values of the phase difference $\alpha$ are the same and can be continuously transformed. There always exists a symmetry operation which consists of the translation for the layer thickness along the layer normal combined with the rotation for a general angle $\alpha$ around the layer normal. The structural parameter phase angle $\alpha$ can be used as a typical parameter which describes the structure. Its value also strongly affects the macroscopic properties of the samples. Since in less recent experiments [2] only macroscopic properties of the materials have been measured, that observations lead to the names of the phases according to their macroscopic properties. Recent experiments [1] have revealed that some phase angles $\alpha$ are typical for these phases, see Table 1 . Temperatures of the phase transitions were measured by differential scanning calorimetry which can detect only first order transitions. Sometimes the temperatures are defined through changes of the texture observations and/or drastical changes of dielectric properties, optical rotatory power (ORP) and others. The transition temperatures are in all mentioned cases more or less unprecise due to the hysteresis or due to the arbitrary 
decision which order of the certain property is strong enough to define the phase. We have to mention again that all transitions are isostructural and therefore the transition temperatures can be observed only for the first order transitions.

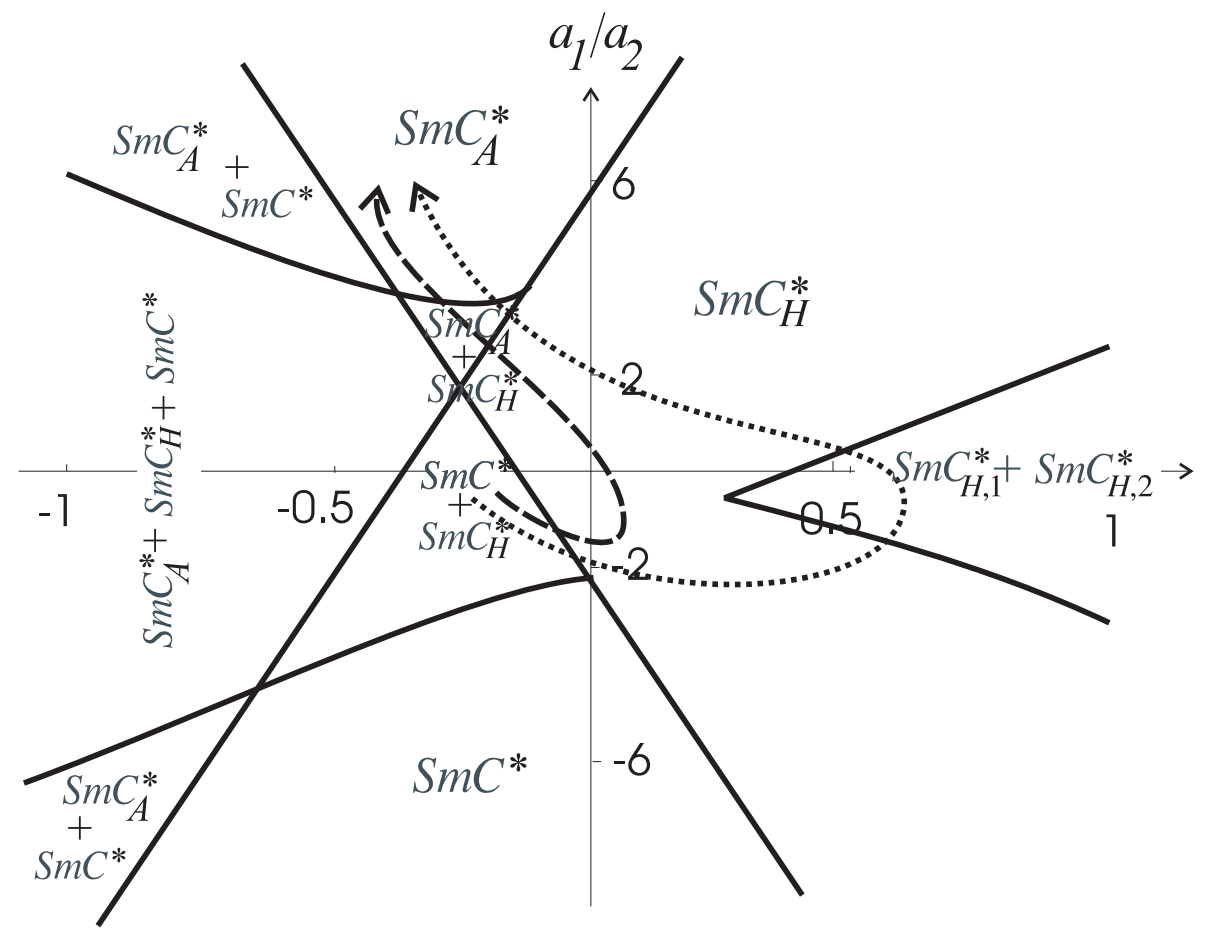

Figure 2: Phase diagram for the ratio $\left|a_{3}\right| / a_{2}=0.2$. A tentative path in the space of model parameters for MHPOBC (dashed line) and the 10OTBBB1M7 (doted line)is given. The arrow marks decreasing temperature.

In the phase diagram (Fig. 2 and 3) we therefore did not distinguish between phases with general angle $\alpha$ or the $\mathrm{Sm}_{H}^{*}$ phase and phases, where the phase angle $\alpha$ is approximately $\pi / 2$ or $2 \pi / 3$, the structures which presumably correspond to the $\mathrm{Sm} \mathrm{C}_{F I 2}^{*}$ and the $\mathrm{Sm} \mathrm{C}_{F I 1}^{*}$ phase, respectively. We used for the ferroelectric $\mathrm{Sm} \mathrm{C}^{*}$ phase and the antiferroelectric $\mathrm{Sm} \mathrm{C}_{A}^{*}$ phase their histor- 


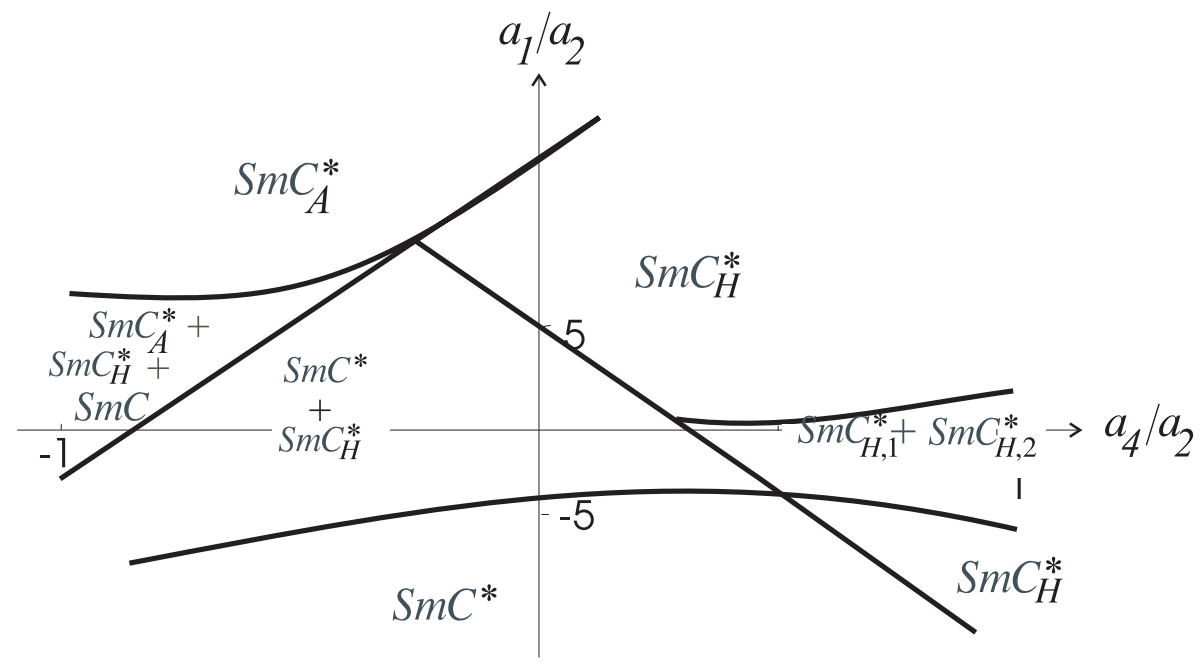

Figure 3: Phase diagram for the ratio $\left|a_{3}\right| / a_{2}=1$.

ical name.

All model parameters change with temperature, since they are the consequence of microscopic interactions which can also change with the temperature because of the changing nematic, smectic and tilt order of the sample as well as the entropical disorder.

The phase diagram of various structures is given for two different ratios of $a_{3} / a_{2}$. The analysis is made only for the competing next nearest neighbors interactions or $a_{2}>0$ and the values of model parameters are expressed in units of $a_{2}$. The value of parameter $a_{3}$ is chosen as a parameter of the phase diagram. The regions where one or more phases are stable, are separated by lines. The stable phases are marked in the region according to the previous discussion. In the region, where the solutions for the two different phase angles $\alpha$ are stable at the same temperatures are marked as the $\mathrm{Sm} \mathrm{C}_{H, 1}^{*}$ and $\mathrm{Sm} \mathrm{C} \mathrm{C}_{H, 2}^{*}$.

The third nearest neighbors coupling is relatively weak in Fig. 
2 and much stronger in Fig. 3. As known also from other systems, the phase diagram for stronger coupling (Fig. 3) is more simple. In general, for strong nearest layers couplings $\left|a_{1} / a_{2}\right|$, larger than other couplings, system always favor either ferroelectric or antiferroelectric ordering regions of the single stable phase $\left(\mathrm{Sm} \mathrm{C}^{*}\right.$ or $\left.\mathrm{Sm} \mathrm{C}_{A}^{*}\right)$. Third nearest neighbors coupling always favors synclinic third nearest neighbors and therefore encourage either simple synclinic ordering or the three layer structures. It therefore introduces first order transition from the ferroelectric $\mathrm{Sm} \mathrm{C}^{*}$ phase to the $\mathrm{Sm} \mathrm{C}_{H}^{*}$ phase as well as broaden the region of the stable $\mathrm{Sm} \mathrm{C}_{H}^{*}$ phases with pitches close to three layers. The result is seen as the expansion of the two phase region $\left(\mathrm{Sm} \mathrm{C} \mathrm{C}^{*}\right.$ and $\mathrm{Sm} \mathrm{C}_{H}^{*}$ ) and the shrinking of the other two phase region ( $\mathrm{Sm} \mathrm{C}_{A}^{*}$ and $\mathrm{Sm} \mathrm{C}_{H}^{*}$ ). The fourth nearest neighbors interactions favor synclinic ordering for its negative sign and therefore favor in the same way $\mathrm{Sm} \mathrm{C}, \mathrm{Sm} \mathrm{C}_{A}^{*}$ as well as the four layers $\mathrm{Sm}_{H}^{*}$. For its large negative values all three phases can be stable for the same temperature. For its positive value it encourages the competition between various interlayer interactions and gives rise to two stable $\mathrm{Sm} \mathrm{C}_{H}^{*}$ phases at the same temperature.

To simulate the behaviour of the most studied antiferroelectric liquid crystal MHPOBC, the parameter changes can follow the path in the parameter space as marked by the dashed line on Fig. 2. The path direction marked by an arrow follows the decreasing of the temperature. When the system becomes tilted, the $\mathrm{Sm} \mathrm{C}_{H}^{*}$ phase is stable. It has a general value od the phase difference $\alpha$ and as such is recognized as the $\mathrm{Sm} \mathrm{C}_{\alpha}^{*}$ phase. With lowering temperature, parameters changes their values and the $\mathrm{Sm} \mathrm{C}^{*}$ phase becomes stable by the first order transition. By cooling again the $\mathrm{Sm} \mathrm{C}_{H}^{*}$ phase with approximately three layers stabilizes, which corresponds to the $\mathrm{Sm} \mathrm{C}_{F I 1}^{*}$ phase and finally the $\mathrm{Sm} \mathrm{C}_{A}^{*}$ phase becomes stable by the first order transition. The parameter path of the material 10OTBBB1M7 studied by x-ray measurements has to go through 
region of the two stable $\mathrm{Sm} \mathrm{C}_{H}^{*}$ phases (dotted line), since two hellicoidally modulated phases separated by the first order transition exist. We have to add that such a parameter path is only tentative, since in real system we have to expect, that it goes through different planes of the $3 \mathrm{D}$ space of model parameters $\left(a_{1} / a_{2}, a_{3} / a_{2}, a_{4} / a_{2}\right)$. To find the real path, also tilt dependence as well as precise phase angle dependence versus temperature, has to be known.

\section{$\underline{\text { CONCLUSIONS }}$}

In this paper we present the phenomenological model with interactions up to the fourth nearest neighbors. We analyze the local stabilities of the synclinic or the ferroelectric Sm C* phase, the anticlinic or the $\mathrm{Sm} \mathrm{C}_{A}^{*}$ phase and the hellicoidal structure with short and in general incommensurate pitch or the $\mathrm{Sm} \mathrm{C}_{H}^{*}$ phase. All structures are in principle of the same symmetry which is defined by symmetry operation consisting from the translation along the layer normal for a layer thickness and rotation for an angle $\alpha$ which can have any value between $-\pi$ and $\pi$. For some special values of the phase difference $\alpha$, the structures has already been known by decades. The phase difference has $\alpha=0$ for the ferroelectric Sm $\mathrm{C}^{*}$ phase, $\alpha=\pi$ for the antiferroelectric phase and has general values in the $\mathrm{Sm} \mathrm{C}_{\alpha}^{*}$ phase. In the $\mathrm{Sm} \mathrm{C}_{F I 2}^{*}$ and the $\mathrm{Sm} \mathrm{C}_{F I 1}^{*}$ phases, the angles are approximately $\pi / 2$ and $2 \pi / 3$, respectively. The last

two structures can therefore be recognized as special cases of the general Sm $\mathrm{C}_{H}^{*}$ structure.

Interactions of longer range induce in the system first order transitions between phases. By the first order transitions, transition temperatures between different phases become observable, since all the transitions are of the isostructural type. We also discuss the arbitrariness of the transition temperatures defined by observations of macroscopic properties, when transitions are continuous. 
In the phase diagram the stability regions of different phases in the model parameters space are presented. Tentative temperature dependence of model parameters is also shown for the two mostly studied materials, MHPOBC and 10OTBBB1M7.

However, in this article only the consequences of interactions of longer range are discussed. Their physical origin is presented in the separate paper [11], where also the physically reasonable magnitudes of the parameters are considered. Within the stability analysis only clock model has been taken into account and possible variations of the phase differences has not been considered and remain as a future problem.

\section{ACKNOWLEDGEMENT}

The financial support of the Ministry of Science of Republic Slovenia is acknowledged.

\section{References}

[1] P. Mach, R. Pindak, A.M. Levelut, P. Barois,H.T. Nguyen, C.C. Huang, L. Furenlid, PRL 81, 1105, (1998).

[2] A. Fukuda, Y. Takanishi, T. Isozaki, K. Ishikawa, H. Takezoe, J. Mater. Chem. 4, 997, (1994) and references therein.

[3] M. Čepič, B. Žekš, Mol. Cryst. Liq. Cryst.,263, 61 (1995).

[4] Ch. Bahr, D. Fliegner, C.J. Booth, J.W. Goodby, PRE 51, R3823 (1995).

[5] B. Rovšek, M. Čepič, B. Žekš, PRE 54, R3113 (1996).

[6] M. Škarabot, M. Čepič, B. Žekš, I. Muševič, G. Heppke, A.V.Kityk, I. Muševič, Phys.Rev.E., $\underline{58}$, 575, (1998).

[7] A. Roy, N.V. Madhusudana, Eur. Phys. J. E, 1, 329 (2000).

[8] M. Čepič, B. Žekš, Mol. Cryst. Liq. Cryst.,301, 221, (1997).

[9] M. Čepič, B. Rovšek, B. Žekš, to appear in Ferroelectrics.

[10] B. Žekš, M. Čepič, Proc. of SPIE 3318, 68 (1998).

[11] M. Čepič, B. Rovšek, B. Žekš, submitted for publication. 